\title{
The Standard-Model Extension and Tests of Relativity $*$
}

\author{
NEIL RUSSELL \\ Physics Department, Northern Michigan University, \\ Marquette, MI 49855, USA \\ nrussell@nmu.edu
}

\begin{abstract}
The Standard-Model Extension, or SME, is a general framework for the study of Lorentz violation in physics. A broad variety of experiments is able to access the SME coefficient space. This proceedings briefly summarizes theory and experiments aimed at testing Special Relativity by measuring these coefficients.
\end{abstract}

Lorentz symmetry is a central feature of the existing theories of gravitation and particle physics. The existence of highly sensitive experiments with the ability to test Lorentz symmetry at unprecedented levels raises the possibility of discovering unconventional effects. This is clearly of interest to physicists since it may pave the way to finding a unified theory of quantum gravity.

A series of publications since 1989 has established a framework, the StandardModel Extension, or SME, that provides a detailed description of possible Lorentz violations in nature in the context of effective field theory. At the basic level, this work focuses on a variety of theoretical issues, including string theory, and spontaneous symmetry breaking. ${ }^{1}$ Much theoretical and experimental effort has been directed towards the study of Lorentz symmetry in Minkowski space, for which the effective field theory is an extension of the Standard Model of particle physics. In flat spacetime, the SME comprises a broad variety of constant coefficients for Lorentz violation that can in principle be measured. ${ }^{2}$ These coefficients transform as conventional Lorentz tensors under observer transformations, but under rotations and boosts of experimental systems, called particle transformations, they are not transformed.

An important category of experimental symmetry tests involves searching for couplings between the electron spin and the Lorentz-violating SME background. The basic idea is that the radiation released in a transition between different spin states has frequency that depends on the spin quantization axis and that differs for particles and antiparticles. Consequently, spectral transitions in atoms with controlled quantization axes, such as occur in atomic clocks, are well suited to tests of Lorentz symmetry. To see small variations in the output frequency of a sensitive clock, one has to compare it to the output of another clock for which the effects are absent, or at least different. So, such experiments are often called clockcomparison experiments. ${ }^{3}$ One of the common scenarios involves monitoring the outputs for long enough to detect the sidereal effects associated with the rotation of the apparatus relative to the distant stars. Tests and theoretical investigations based on these ideas include ones done for hydrogen masers, antihydrogen, noble-gas masers, space-mounted atomic clocks, Penning traps, and torsion pendula. ${ }^{4}$

*Proceedings of 11th Marcel Grossmann Meeting, Berlin, Germany, 23-29 July, 2006. 
The effects of Lorentz-violation on the electromagnetic sector are described by 19 coefficients at leading order and are amenable to sensitive experimental investigations. Analysis of birefringence data from cosmological sources has placed stringent limits on 10 of these, while optical and microwave cavity resonators have placed limits on the remaining ones. ${ }^{5}$ Cosmological birefringence tests are based on distant processes producing the radiation, but offer fantastic sensitivities. Laboratory cavity experiments have undergone numerous innovations to improve their experimental reach, including cryogenic cooling, the use of optical sapphire crystals, and placement on rotating turntables to exploit geometrical properties. Other investigations involving photons include, for example, ones based on Cerenkov radiation, synchrotron radiation, Compton scattering, and Doppler-shift experiments. ${ }^{6}$

Lorentz symmetry has also been tested in the context of various other particles. For example, in the case of neutrinos, simple models constructed from the SME coefficients have been found to be consistent with known neutrino data while offering the advantage of fewer parameters and masses. ${ }^{7}$ Accelerator-related physics investigations of Lorentz symmetry include ones with a variety of neutral mesons and others with muons. ${ }^{8}$ Further details of Lorentz tests in flat spacetime can be found in various overview sources. ${ }^{9}$

The gravitational sector of the Standard-Model Extension consists of a framework for addressing Lorentz and CPT violation in curved spacetimes, including ones with torsion. ${ }^{10}$ The coefficients for Lorentz violation typically vary with position, adding complexity to the manner in which matter couples to the background. To set up the framework for the full Standard-Model Extension, the vierbein formalism can be adopted, since it allows the spinor properties of ordinary matter to be incorporated. It also has the useful feature of distinguishing naturally between local Lorentz transformations and general coordinate transformations. Lorentz symmetry breaking must be either explicit or spontaneous. A study of this topic has shown that explicit Lorentz violation, in which the breaking occurs in the Lagrangian density, is incompatible with generic Riemann-Cartan spacetimes. On the other hand, spontaneous breaking can be successfully introduced in a consistent manner. One of the far-reaching results associated with spontaneous Lorentz breaking is that it always goes hand in hand with spontaneous breaking of diffeomorphism symmetry. The 10 possible Nambu-Goldstone modes associated with the six generators for Lorentz transformations and the four generators for diffeomorphisms have been studied. The fate of these modes depends on the spacetime geometry and the dynamics of the tensor field triggering the spontaneous Lorentz violation. The results are consistent with the known massless particles in nature, the photon and the graviton. An extensive study has been made of the pure-gravity sector of the SME with the aim of finding possible experimental consequences. Of particular interest are experiments involving lunar and satellite laser ranging, laboratory tests with gravimeters and torsion pendula, measurements of the spin precession of orbiting gyroscopes, timing studies of signals from binary pulsars, and the classic tests involving the perihelion precession and the time delay of light. The sensitivity range of these experiments is 
parts in $10^{4}$ to parts in $10^{15}$.

\section{References}

1. V.A. Kostelecký and R. Potting, Phys. Rev. D 51, 3923 (1995); Phys. Lett. B 381, 89 (1996); Phys. Rev. D 63, 046007 (2001); Nucl. Phys. B 359, 545 (1991); V.A. Kostelecký and S. Samuel, Phys. Rev. D 39, 683 (1989); Phys. Rev. Lett. 63, 224 (1989); Phys. Rev. D 40, 1886 (1989); Phys. Rev. Lett. 66, 1811 (1991); B. Altschul and V.A. Kostelecký, Phys. Lett. B 628, 106 (2005).

2. D. Colladay and V.A. Kostelecký, Phys. Rev. D 55, 6760 (1997); Phys. Rev. D 58, 116002 (1998); V.A. Kostelecký and R. Lehnert, Phys. Rev. D 63, 065008 (2001).

3. V.A. Kostelecký and C.D. Lane, Phys. Rev. D 60, 116010 (1999); P. Wolf et al., Phys. Rev. Lett. 96, 060801 (2006); F. Cane et al., Phys. Rev. Lett. 93, 230801 (2004); D.F. Phillips et al., Phys. Rev. D 63, 111101 (2001); M.A. Humphrey et al., Phys. Rev. A 68, 063807 (2003); Phys. Rev. A62, 063405 (2000); D. Bear et al., Phys. Rev. Lett. 85, 5038 (2000).

4. G.M. Shore, Nucl. Phys. B 717, 86 (2005); R. Bluhm et al., Phys. Rev. Lett. 82, 2254 (1999); Phys. Rev. Lett. 88, 090801 (2002); Phys. Rev. D 68, 125008 (2003); Phys. Rev. Lett. 79, 1432 (1997); Phys. Rev. D 57, 3932 (1998); H. Dehmelt et al., Phys. Rev. Lett. 83, 4694 (1999); R.K. Mittleman et al., Phys. Rev. Lett. 83, 2116 (1999); G. Gabrielse et al., Phys. Rev. Lett. 82, 3198 (1999); R. Bluhm and V.A. Kostelecký, Phys. Rev. Lett. 84, 1381 (2000); B.R. Heckel et al., Phys. Rev. Lett. 97, 021603 (2006); L.-S. Hou et al., Phys. Rev. Lett. 90, 201101 (2003); D. Colladay and P. McDonald, Phys. Rev. D 73, 105006 (2006).

5. S. Herrmann et al., Phys. Rev. Lett. 95, 150401 (2005); P.L. Stanwix et al., Phys. Rev. Lett. 95, 040404 (2005); P. Antonini et al., Phys. Rev. A 71, 050101 (2005); Phys. Rev. A 72, 066102 (2005); M.E. Tobar et al., Phys. Rev. D 71, 025004 (2005); Phys. Rev. A 72, 066101 (2005); P. Wolf et al., Phys. Rev. D 70, 051902 (2004); Gen. Rel. Grav. 36, 2352 (2004); H. Müller et al., Phys. Rev. D 68, 116006 (2003); Phys. Rev. Lett. 91020401 (2003); J.A. Lipa et al., Phys. Rev. Lett. 90, 060403 (2003); V.A. Kostelecký and M. Mewes, Phys. Rev. Lett. 87, 251304 (2001); Phys. Rev. D 66, 056005 (2002); Phys. Rev. Lett. 97, 140401 (2006).

6. R. Lehnert and R. Potting, Phys. Rev. Lett. 93, 110402 (2004); Phys. Rev. D 70, 125010 (2004); B. Altschul, Phys. Rev. Lett. 96, 201101 (2006); Phys. Rev. D 74, 083003 (2006); C.D. Lane, Phys. Rev. D 72, 016005 (2005).

7. V.A. Kostelecký and M. Mewes, Phys. Rev. D 69, 016005 (2004); Phys. Rev. D 70, 031902 (2004); Phys. Rev. D 70, 076002 (2004); T. Katori et al., Phys. Rev. D 74, 105009 (2006); LSND Collab., Phys. Rev. D 72, 076004 (2005).

8. OPAL Collab., Z. Phys. C 76, 401 (1997); BABAR Collab., hep-ex/0607103 FOCUS Collab., Phys. Lett. B 556, 7 (2003); V.A. Kostelecký, Phys. Rev. Lett. 80, 1818 (1998); Phys. Rev. D 61, 016002 (2000); Phys. Rev. D 64, 076001 (2001); D. Colladay and V.A. Kostelecký, Phys. Lett. B 344, 259 (1995); Phys. Rev. D 52, 6224 (1995); V.A. Kostelecký and R. Van Kooten, Phys. Rev. D 54, 5585 (1996); N. Isgur et al., Phys. Lett. B 515, 333 (2001); R. Bluhm, V.A. Kostelecký and C.D. Lane, Phys. Rev. Lett. 84, 1098 (2000); V.W. Hughes et al., Phys. Rev. Lett. 87, 111804 (2001); Muon g-2 Collab., hep-ex/0110044

9. R. Bluhm, arXiv:hep-ph/0506054 D. Mattingly, Living Rev. Rel. 8, 5 (2005).

10. V.A. Kostelecký, Phys. Rev. D 69, 105009 (2004); R. Bluhm and V.A. Kostelecký, Phys. Rev. D 71, 065008 (2005); V.A. Kostelecký and R. Potting, Gen. Rel. Grav. 37, 1675 (2005); Q.G. Bailey and V.A. Kostelecký, Phys. Rev. D 74, 045001 (2006). 